\title{
Coordinated Two-Stage Volt/Var Management in Distribution Networks
}

\author{
Lesiba Mokgonyana ${ }^{a, *}$, Jiangfeng Zhang ${ }^{\mathrm{a}, \mathrm{b}}$, Lijun Zhang ${ }^{\mathrm{a}}$, Xiaohua Xia ${ }^{\mathrm{a}}$ \\ ${ }^{a}$ Department of Electrical, Electronic and Computer Engineering, University of Pretoria, Pretoria \\ 0002, South Africa \\ ${ }^{b}$ Department of Electronic and Electrical Engineering, University of Strathclyde, Glasgow G1 $1 X W$, \\ $U K$
}

\begin{abstract}
This paper investigates daily volt/var control in distribution networks using feeder capacitors as well as substation capacitors paired with on-load tap changers. A twostage coordinated approach is proposed. Firstly, the feeder capacitor dispatch schedule is determined based on reactive power heuristics. Then, an optimisation model is applied to determine the dispatch schedule of the substation devices taking into account the control actions of the feeder capacitors. The reference voltage of the substation secondary bus and the tap position limits of transformers are modified such that the model adapts to varying load conditions. The optimisation model is solved with a modified particle swarm optimisation algorithm. Furthermore, the proposed method is compared with conventional volt/var control strategies using a distribution network case study. It is demonstrated that the proposed approach performs better than the conventional strategies in terms of voltage deviation and energy loss minimisation.

Keywords: Capacitor; Distribution network; Energy loss; On-load tap changer;

Particle swarm optimisation; Volt/var control.
\end{abstract}

\section{Introduction}

Control of volt and var devices such as on-load tap changers (OLTC) and shunt capacitors affects the voltage profile and the total power loss in distribution networks[1].

\footnotetext{
*Corresponding author

Email address: u24062147@tuks.co.za (Lesiba Mokgonyana)
} 
Dispatch of the volt/var control (VVC) resources can be performed in a coordinated 5 manner in constrained environments to meet specific operational objectives [2, 3, 4, 5, 6, 7, 8, 9, 10, 11, 12, 13, 14, 15, 16, 17]. The complexity of the objective function, constraints, and computation is influenced by, among others: regulatory limits, switching limitations and available control devices. The focus of this paper is daily VVC with switching restrictions, which is usually applied to networks with widespread communication and control coverage [7, 8, 9, 10, 11, 12, 13, 14, 15, 16, 17]. The additional requirement of this VVC approach is a day-ahead forecast of load behaviour, which is made possible by the existence of load forecasting techniques that provide good accuracy [18], [19].

Daily coordinated control of all distribution devices is computationally complex, but there are a number of ways to deal with this difficulty. One way to approach this is to simplify the solution space so as to reduce the computational burden. For instance, the requirements of dynamic programming can be eased according to [8], [9], [11], while a more efficient solver based on the interior-point method is presented in [17]. In [10] the number of possible states is decreased by combining artificial neural networks, a rulebased method and dynamic programming. Use of heuristic rules can also reduce the number of possible device operations, therefore simplifying the optimisation model [13]. These methods address the issue of computational complexity but the requirements for remote control infrastructure remain for network-wide implementation. Another alternative is to divide the scheduling problem into two sub-problems: one handling the dispatch of the substation capacitor (SC) and OLTC, and the other controlling the feeder capacitors (FCs) [12]. In particular, dispatch of the substation devices minimises reactive power-flow and the voltage deviation at the substation bus. FCs are then dispatched based on local bus voltage and power factor deviations using a fuzzy control scheme after the substation devices have been dispatched. The final states of the FCs are found when the bus voltage is within permissible limits. In [20] the total loss and voltage deviations at load buses are minimised through dispatch of all capacitors. The OLTC is controlled in real-time to keep the substation secondary bus voltage close to 
the set-point that incorporates the voltage change caused by the capacitors. In these previous approaches, the objectives specified for the substation control problem focus only on the secondary bus at the substation. The rest of the buses are considered in the control schemes for capacitors.

In this paper, a two-stage approach to daily VVC is presented with the devices controlled in a different manner. Firstly, a strategy to determine the FC dispatch schedule is developed using reactive power set-points. Then, with the FC schedule as input, coordination of the SC and OLTC is formulated as an optimisation problem. The advantage of this approach is that the voltage deviations can be reduced further by adjusting the transformer tap ratio together with the capacitor on/off statuses. The reason is that, unlike capacitors, transformers equipped with OLTCs usually have a larger control range, smaller discrete steps and provide direct voltage adjustments. Hence the model can produce an improved voltage profile. Voltage magnitudes at all load buses are primarily controlled at the substation with the statuses of FCs given as input. Since the voltage constraint at each load bus is handled at the substation, the FC control problem focuses solely on loss reduction. This strategy facilitates the adoption of a technique relying only on reactive power-flows at the substation to determine the control actions for FCs. The FC control problem minimises the reactive powerflow through the distribution feeders at the substation bus, while the OLTC and SC problem minimises both the total loss and the voltage deviations at all distribution network buses. The decomposition of the VVC problem reduces the dimension of the optimisation model. Furthermore, the model can be applied to networks with extensive remote control capability and those with limited capability i.e. coverage from the control centre to the substation but no coverage along medium voltage distribution lines.

The FC control problem is solved with a heuristic method while the OLTC and SC control problem is solved by particle swarm optimisation (PSO) with consideration of the discrete nature of the control variables. The performance of the proposed approach 60 is analysed in relation to various implementations of conventional VVC. Simulation results show that the proposed approach minimises both the voltage deviations and the 
total energy loss while conventional control considers one objective at a time depending on specified settings.

The remainder of this paper is structured as follows. Section 2 gives a brief introduction of conventional VVC methods. Section 3 presents the proposed control strategy. A case study is described, followed by a discussion of results in Section 4. Section 5 concludes the study.

\section{Conventional VVC}

In traditional distribution networks, voltage regulation is realised through manual or automatic adjustments of transformer taps so that the voltage lies between the given upper and lower bounds. Automatic voltage regulation is achieved by using transformers equipped with OLTCs at distribution substations. Generally, automatic voltage regulation (AVR) settings comprise a voltage set-point and a deadband. The voltage set-point is the desired voltage at the bus controlled by the OLTC. The deadband is the allowed margin within which no tap changes are initiated; the controller sends out tap changes whenever the voltage falls outside the deadband. The steady-state tap position at time $t$ is determined using [21]

$$
u_{\mathrm{TAP}}^{t}= \begin{cases}u_{\mathrm{TAP}}^{t-1}+\Delta u_{\mathrm{TAP}}^{t}, & \text { if } V_{\mathrm{set}}-V^{t}>0.5 V_{\mathrm{db}} \\ u_{\mathrm{TAP}}^{t-1}-\Delta u_{\mathrm{TAP}}^{t}, & \text { if } V_{\mathrm{set}}-V^{t}<-0.5 V_{\mathrm{db}} \\ u_{\mathrm{TAP}}^{t-1}, & \text { otherwise; }\end{cases}
$$

where $\Delta u_{\text {TAP }}^{t}$ is the number of tap movements required to bring the voltage $V^{t}$ into the deadband, $V_{\mathrm{db}} ; V_{\text {set }}$ is the voltage set-point. The deadband setting is, in general, selected in a way that avoids unnecessary operations [22]. The voltage set-point decision considers feeder losses and voltage regulation limits [23].

Capacitor control aims to reduce reactive power-flow through the substation transformer or the distribution feeder. In this way, the total loss is minimised. The use 75 of capacitors also has the effect of raising voltage in addition to providing reactive 


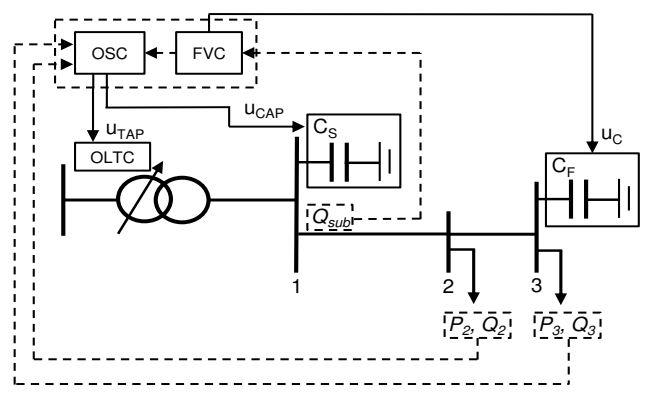

Fig. 1. Conceptual model of the proposed feeder var control and optimal substation control.

power compensation. In this paper, a combination of automatic voltage regulation using OLTCs and time-based capacitor control is implemented. This method requires no remote control facilities because the OLTC and capacitors are controlled locally. It is henceforth referred to as conventional control.

\section{Feeder Var Control and Optimal Substation Control (FVC-OSC)}

The proposed method minimises the total loss and voltage deviations in distribution systems. The conceptual model of the method is illustrated in Fig. 1, The solution process comprises two stages. Firstly the FC schedules are determined. Then the SC and OLTC are coordinated optimally to complete the dispatch schedules. The FC on/off statuses are derived from reactive power variations on the feeder at the substation and supplied together with the forecasted active power and reactive power profiles of the loads to the SC and OLTC control model. Then, an optimisation algorithm is applied in which the switching sequences of the SC and OLTC are obtained.

The index $t$ denotes a time interval, whose length is $\Delta t=1 \mathrm{~h} ;[t \Delta t,(t+1) \Delta t)$ denotes a single interval and $N$ specifies the total number of intervals in the scheduling period, which in this study is 24 . The time interval and bus indices satisfy $1 \leq t \leq N$, $1 \leq d \leq D$ and $1 \leq j \leq D$. $D$ is the total number of buses in a given network. The transformer tap position (adjusted by an OLTC) is represented by the integer variable $u_{\mathrm{TAP}}^{t} \in\left[T^{\min }, T^{\max }\right]$ and related to the tap ratio $a_{r}^{t}$ by $a_{r}^{t}=1-u_{s t} u_{\mathrm{TAP}}^{t} \cdot u_{s t}$ is the size of a single tap increment/decrement step. Capacitors are realised as shunt elements with 
on/off operations modeled as binary variables. $u_{\mathrm{CAP}}^{t} \in[0,1]$ denotes the SC control action while the FC control actions are represented by $u_{\mathrm{C}, d}^{t} \in[0,1]$.

\subsection{Feeder Var Control (FVC)}

A heuristic scheduling technique based on the substation feeder reactive power profiles is proposed for FVC. The aim of this method is to minimise reactive power-flow through the transformer without SC and OLTC control. Furthermore, the total number of operations for the FCs during the scheduling period must not exceed the permissible limit.

The objective of FVC is to minimise feeder reactive power-flow as described below:

$$
\sum_{t=1}^{N}\left|Q_{\mathrm{sub}, n}^{t}\right|
$$

subject to

105 (1) power-flow balance at switching interval $t$ :

$$
\begin{array}{r}
P_{\mathrm{G}, d}^{t}-P_{\mathrm{L}, d}^{t}=V_{d}^{t} \sum_{j=1}^{D} V_{j}^{t}\left[G_{d j}^{0} \cos \left(\delta_{d}^{t}-\delta_{j}^{t}\right)\right. \\
\left.+B_{d j}^{0} \sin \left(\delta_{d}^{t}-\delta_{j}^{t}\right)\right], \\
Q_{\mathrm{G}, d}^{t}-Q_{\mathrm{L}, d}^{t}=V_{d}^{t} \sum_{j=1}^{D} V_{j}^{t}\left[G_{d j}^{0} \sin \left(\delta_{d}^{t}-\delta_{j}^{t}\right)\right. \\
\left.-B_{d j}^{0} \cos \left(\delta_{d}^{t}-\delta_{j}^{t}\right)\right],
\end{array}
$$

(2) FC switching effort:

$$
\sum_{t=2}^{N}\left|u_{\mathrm{C}, d}^{t}-u_{\mathrm{C}, d}^{t-1}\right| \leq C^{\max }
$$

where $Q_{\mathrm{sub}, n}^{t}$ is the reactive power through the distribution feeder $n$ at the substation. $P_{\mathrm{G}, d}^{t}$ denotes the generated active power and $Q_{\mathrm{G}, d}^{t}$, the generated reactive power, at bus $d$. $P_{\mathrm{L}, d}^{t}$ and $Q_{\mathrm{L}, d}^{t}$ stand for the active power and reactive power consumed at the 
$d$ th bus respectively. $V_{d}^{t}$ and $\delta_{d}^{t}$ are the voltage magnitude and angle at bus $d$; at bus $j$, the voltage magnitude and angle are denoted by $V_{j}^{t}$ and $\delta_{j}^{t}$. $G_{d j}^{0}$ and $B_{d j}^{0}$ are the conductance and susceptance values of the admittance element $y_{d j}^{0}$ in the admittance matrix, respectively, when the status of the SC is off and the transformer tap position is at nominal tap. $C^{\max }$ indicates the allowable maximum number of daily $\mathrm{FC}$ switching operations.

\subsection{Calculation of the FC Switching Sequence}

The FCs are switched on/off if the resulting status decreases the reactive power flowing through the distribution feeders. The control actions of the FCs at interval $t$ are calculated using

$$
u_{\mathrm{C}, d}^{t}= \begin{cases}1, & \text { if } Q_{\mathrm{seg}, n}^{t} \geq z_{1} Q_{d}^{\mathrm{F}} \\ 0, & \text { if } Q_{\mathrm{seg}, n}^{t} \leq z_{2} Q_{d}^{\mathrm{F}} \\ u_{\mathrm{C}, d}^{t-1}, & \text { if } z_{2} Q_{d}^{\mathrm{F}}<Q_{\mathrm{seg}, n}^{t}<z_{1} Q_{d}^{\mathrm{F}}\end{cases}
$$

where $Q_{\mathrm{seg}, n}^{t}$ is the reactive power-flow at the head of the lateral or segment where the FC is connected. For a feeder that does not have laterals, $Q_{\mathrm{seg}, n}^{t}=Q_{\mathrm{sub}, n}^{t} . Q_{d}^{\mathrm{F}}$ is reactive power rating of the capacitor. $z_{1}$ and $z_{2}$ are switching parameters selected based on the feeder reactive power-flows at the substation. $z_{2}$ is smaller than $z_{1}$ and the two parameters satisfy $0<z_{1} \leq 1$ and $-1 \leq z_{2}<0$.

The operation sequence of FCs are assigned locational priority and their operation is determined sequentially using (5). The switch status of the capacitor furthest from the substation, is determined first whereas that of the nearest capacitor is last in line. The resulting capacitor statuses affect bus voltage magnitudes, but it is not necessary to consider voltage regulation in (11)-(5) because the bus voltages are kept within the upper and lower limits by the SC and OLTC control described in Section 3.3 . 


\subsection{Optimal Substation Control (OSC)}

The total energy loss and the secondary side voltage deviations minimisation problem can be formulated as:

$$
J_{1}(\boldsymbol{u}, \boldsymbol{x})+J_{2}(\boldsymbol{u}, \boldsymbol{x})=\lambda_{1} \sum_{t=1}^{N}\left(\left(V_{R}-V_{1}^{t}\right)^{2}+\sum_{d=2}^{D}\left(V_{d}^{\mathrm{T}}-V_{d}^{t}\right)^{2}\right)+\lambda_{2} \sum_{t=1}^{N} P_{\text {loss }}^{t},
$$

$130 \quad$ subject to

(1) bus voltage limits:

$$
V^{\min } \leq V_{d}^{t} \leq V^{\max }
$$

(2) power-flow balance at interval $t$ : (2) and (3) apply except that $G_{d j}^{0}$ and $B_{d j}^{0}$ are replaced with $G_{d j}^{i}$ and $B_{d j}^{i}$,

(3) OLTC control movements:

$$
\sum_{t=2}^{N}\left|u_{\mathrm{TAP}}^{t}-u_{\mathrm{TAP}}^{t-1}\right| \leq u_{T}^{\max }
$$

(4) and SC switching operations, which are taken into account using the same expression as (4);

where the first term in (66) represents the voltage deviation index (VDI) and the second term the total energy loss; $\lambda_{1}$ and $\lambda_{2}$ are positive tuning weights; $V_{1}^{t}$ is voltage magnitude at the substation secondary bus and $V_{R}$ denotes the reference voltage at the substation secondary bus which satisfies $V_{R}^{\min } \leq V_{R} \leq V_{R}^{\max } ; V_{R}^{\min }$ and $V_{R}^{\max }$ are the lower and upper reference voltage limits respectively; $V_{d}^{t}$ is the voltage magnitude at bus $d ; V_{d}^{\mathrm{T}}$ is the target voltage at bus $d ; V^{\text {min }}$ and $V^{\text {max }}$ are the permissible minimum and maximum voltages respectively; $u_{T}^{\max }$ is the allowed maximum number of tap-changing operations in a day.

Some tap positions within the allowable range do not produce feasible bus voltages under time-varying loading conditions. Therefore, the search space is reduced by further narrowing the tap range from $\left[T^{\min }, T^{\max }\right]$ to probable tap positions that lead to the 
lowest voltage deviations. Using the expression derived in[8], the ideal tap ratio is first approximated as

$$
a_{\mathrm{id}}^{t}=\left\{\frac{\left|Z_{\mathrm{T}}\right|^{2}}{\left|V_{\mathrm{p}}^{t}\right|^{2}\left|V_{\mathrm{s}}^{t}\right|^{2}}\left[\left(Q_{\mathrm{L}}^{t}+\frac{\left|V_{\mathrm{s}}^{t}\right|^{2}}{\left|Z_{\mathrm{T}}\right|}\right)^{2}+P_{\mathrm{L}}^{t^{2}}\right]\right\}^{\frac{1}{2}}
$$

then the tap position range is modified as follows:

$$
\frac{a_{\mathrm{id}}^{t}-1}{u_{s t}}-u_{a} \leq u_{\mathrm{TAP}}^{t} \leq \frac{a_{\mathrm{id}}^{t}-1}{u_{s t}}+u_{a}
$$

where $\boldsymbol{u}$ represents a vector containing control variables that consist of time-varying tap positions and capacitor switching statuses; $\boldsymbol{x}$ is a vector containing state variables; $V_{\mathrm{p}}^{t}$ is the substation primary bus voltage whereas $V_{\mathrm{s}}^{t}$ represents the substation secondary bus voltage; $Z_{\mathrm{T}}$ is the impedance of the transformer; $P_{\mathrm{L}}^{t}$ and $Q_{\mathrm{L}}^{t}$ denote the active and reactive power demand at the substation secondary bus respectively; $u_{a}$ is a positive integer.

\subsection{OSC Solution Algorithm}

The solution algorithm presented in this section solves the problem described in (6) (10). Since the standard PSO addresses continuous problems, it is modified in the proposed approach to cater for discrete variables. The resulting algorithm is similar to that presented in [24]. The particle $p$ position vector is represented by $\boldsymbol{x}_{p}=\left[x_{p}^{1}, x_{p}^{2}, \ldots, x_{p}^{U}\right]^{T}$, where $U$ is the particle dimension. The velocity vector is $\boldsymbol{v}_{p}=\left[v_{p}^{1}, v_{p}^{2}, \ldots, v_{p}^{U}\right]^{T}$. The position and velocity for particle $p$ in the next iteration, $k+1$, are updated as follows

$$
\begin{gathered}
\boldsymbol{x}_{p}^{k+1}=\boldsymbol{x}_{p}^{k}+\boldsymbol{v}_{p}^{k+1}, \\
\boldsymbol{v}_{p}^{k+1}=\operatorname{round}\left(w \boldsymbol{v}_{p}^{k}+c_{1} \operatorname{rand}_{1}\left(\boldsymbol{x}_{1 p}^{k}-\boldsymbol{x}_{p}^{k}\right)+c_{2} \operatorname{rand}_{2}\left(\boldsymbol{x}_{1 g}^{k}-\boldsymbol{x}_{p}^{k}\right)\right),
\end{gathered}
$$

where round () indicates that the result is rounded off to the nearest discrete value; $x_{1 p}^{k}$ (pbest) is the best position of particle $p$, and $\boldsymbol{x}_{1 g}^{k}$ (gbest) is the best position of the whole particle group; $c_{1}$ and $c_{2}$ are acceleration constants which satisfy $c_{1}+c_{2}=4$; 
rand $_{1}$ and rand $_{2}$ are random numbers between 0 and 1 . The velocity $\boldsymbol{v}_{p}^{k}$ is restricted to fall within the range $\left[v^{\min }, v^{\max }\right]$. When $v_{p}^{u}<v^{\min }$, the velocity is reset to $v_{p}^{u}=v^{\min }$ in all iterations. For cases where $v_{p}^{u}>v^{\max }$, the velocity is redefined to $v_{p}^{u}=v^{\max }$. As is the case with velocities, the particle position $\boldsymbol{x}_{p}^{k}$ is bounded by $x^{\min }$ and $x^{\max }$. To deal with the constraints (7)-(44), the penalty approach described in (13) is implemented

$$
J_{1}(\boldsymbol{u}, \boldsymbol{x})+J_{2}(\boldsymbol{u}, \boldsymbol{x})+p \sum_{u=1}^{M} \max \left(0, h_{u}\right),
$$

where $p$ is the penalty coefficient. $J_{1}$ and $J_{2}$ are defined in 6. $h_{u}$ is an expression representing each of the constraints. $M$ is the total number of constraints. The value of the penalty term in (13) rises as the search moves into infeasible regions and vice versa.

The following PSO parameter settings are employed: particle population size of 100; acceleration constants are set as $c_{1}=c_{2}=2$; the inertia term, $w=0.5+1 / 2(\ln k+1)$, where $k$ is the iteration count. $v^{\max }$ is set at about $25 \%$ of the variable range; $v^{\min }=$ $-v^{\max }$.

\subsection{FVC-OSC Flowchart}

FVC-OSC is based on a low-complexity approach to VVC. Consider a system with four FCs, one SC and one OLTC for a scheduling period of $24 \mathrm{~h}$. A heuristic technique is used to schedule FCs, leaving the dispatch schedule of the SC and OLTC to be solved optimally. The OSC variables are the $\mathrm{SC}$ on/off statuses, transformer tap positions and the reference voltage at the substation secondary bus. Thus, the total number of variables is $(1+1 \times 24+1 \times 24)=49$. For a unified model, the dispatch schedule of the OLTC and all capacitors are calculated simultaneously. One such example is the dynamic programming approach developed in [11], which increases the number of variables to $(5 \times 24+1 \times 24)=144$.

Furthermore, the proposed model is suitable for networks with extensive control capability and those with remote control at the substation but not downstream. The latter may even be a case of intentional selective upgrading of existing infrastructure. 
This is preferred when the transition to a fully automated network is not possible because of the prohibitive cost of upgrades [25], [26]. Since FVC is an independent form of control, it can be implemented in a similar manner to conventional time control. Implementation of the FVC-OSC model in a distribution network incapable of remote FVC would require a local controller, which would be loaded with hourly control actions.

The procedure that is followed to solve the VVC problem is summarised below and illustrated in Fig. 2,

(1) Solve the power-flow problem for all $t=1,2,3, \ldots, N$ and determine a set of control actions $u_{\mathrm{C}, d}^{t}$ according to (1)-(5) over the 24-h scheduling period.

(2) Determine the applicable transformer tap range for each interval $t$.

(3) Initialise all variables $\left(u_{\mathrm{CAP}}^{t}, u_{\mathrm{TAP}}^{t}\right.$ and $\left.V_{R}\right)$ for all $t=1,2,3, \ldots, N$.

(4) Solve the power-flow equations to determine the bus voltages and power losses for a given population of particles. At the end of the power-flow computation for interval $t$, the vector $\left[V_{1}^{t}, \ldots, V_{d}^{t}\right]^{T}$ and $P_{\text {loss }}^{t}$ are stored until the end of the scheduling period and then transferred to the next step where the optimisation model is evaluated.

(5) Apply discrete PSO.

(6) Stop the algorithm if it has reached the predefined maximum number of iterations. Otherwise return to step 4.

\section{Case Study and Results Discussion}

The distribution network depicted in Fig. 3 is used for performance evaluation of conventional control, the optimum settings approach (OSA) and FVC-OSC. Full details of the network can be found in [27], [28]. The demand at each load bus peaks at 1.4 MVA and has the $P_{\mathrm{L}, d}^{t}$ and $Q_{\mathrm{L}, d}^{t}$ profiles shown in Fig. 因for which $\Delta t=1 \mathrm{~h}$. The minimum and maximum allowable voltages are $0.95 \mathrm{pu}$ and $1.05 \mathrm{pu}$ respectively. The target voltage is $V_{d}^{\mathrm{T}}=1 \mathrm{pu}$ for all load buses. The transformer provides $\pm 10 \%$ regulation with 8 tap steps above and below nominal tap; $T^{\min }=-8$ and $T^{\max }=8$. Each step gives an increment/decrement of $\pm 0.0125 \mathrm{pu}$. The maximum allowable number of tap operations 


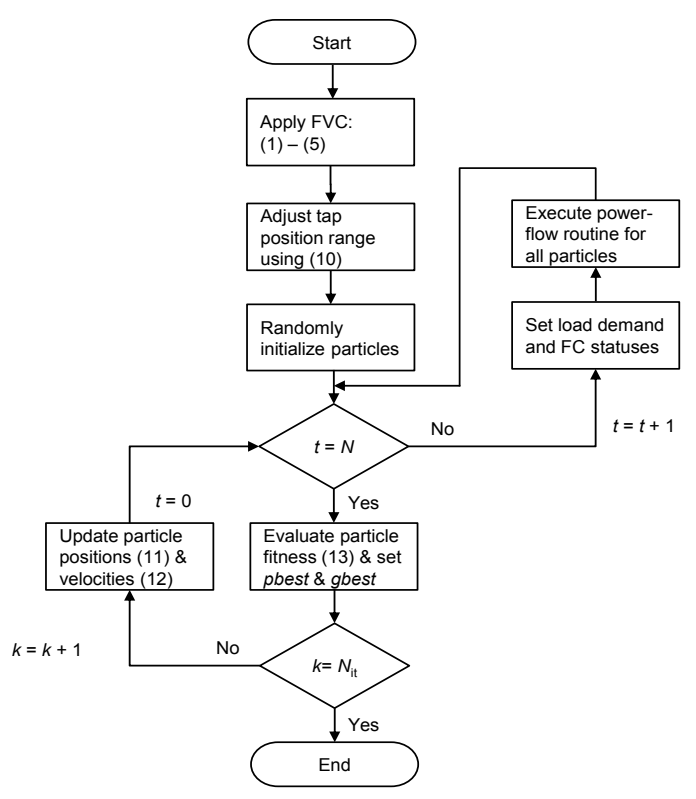

Fig. 2. FVC-OSC flowchart. $N_{\text {it }}$ is iteration limit.

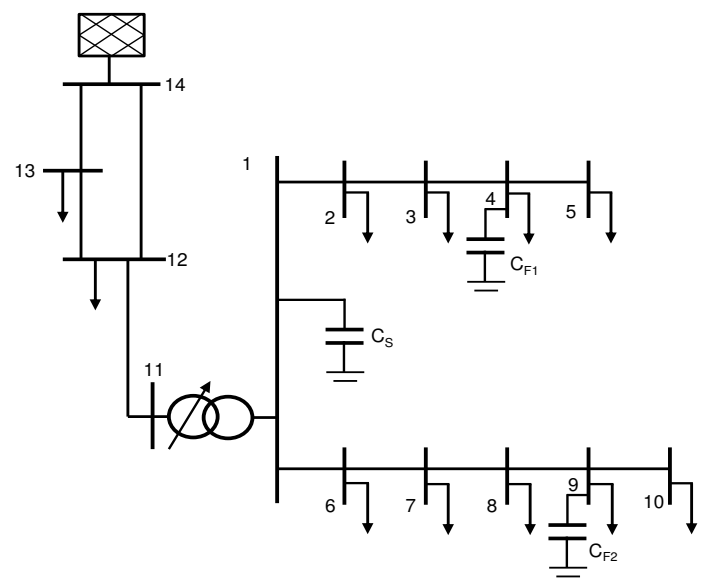

Fig. 3. Typical distribution network with an OLTC and capacitors (adapted from [27]). 


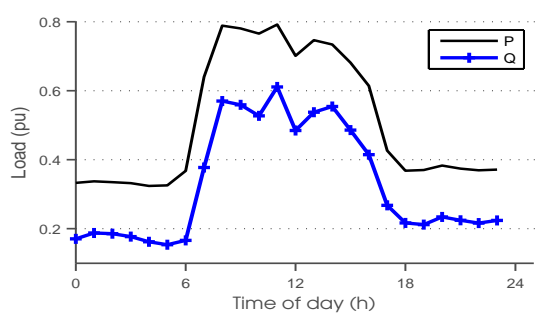

Fig. 4. Daily load curves.

per day is 30. All capacitors can only be operated 8 times a day at the most. Two cases of conventional control, CC-A and CC-B, which refer to AVR combined with time-based capacitor control at different set-points $\left(V_{\text {set }}\right)$ are investigated for comparison purposes. The settings used for conventional control are shown in Table 1. For both CC-A and CC-B, the SC is switched on, at 07:00, as the load rises towards peak demand and switched off, at 16:00, as the load approaches minimum demand.

OSA introduces some improvement to conventional control. As described in [27], these settings are derived from heuristic rules which aim to coordinate the OLTC, SC and FCs to minimise losses and satisfy the bus voltage constraint. In brief, losses in a traditional distribution network without distributed generation can be minimised by making $V_{\text {set }}$ high but below $V^{\max }$ and selecting $V_{\mathrm{db}}$ in a way that keeps the actual voltage close to $V_{\text {set }}$ but does not cause too many tap movements. The SC set-points, $Q_{\text {on }}$ and $Q_{\text {off }}$, are based on the reactive power-flow through the substation transformer. The FC set-points denoted by $V_{\text {on }}$ and $V_{\text {off }}$ depend on the bus voltage thresholds at the point of connection. Here, $V_{\mathrm{db}}$ is selected such that the number of tap movements does not exceed $u_{T}^{\max }$. The same settings as in [27] are used in this case study as shown in Table 2 ,

Regarding the proposed FVC-OSC approach, $z_{1}=0.5$ and $z_{2}=-z_{1}$ are used for FC control. The power-flow solutions are computed using Newton's method provided in [29]. In terms of computational times, FVC-OSC takes 13 minutes to execute, including 100 iterations of PSO on a $3.3 \mathrm{GHz}$ Intel i5-4590 (8 GB RAM) computer. The time requirement increases to 26 minutes, to run the algorithm with 200 PSO iterations 
Table 1

Conventional Method Settings

\begin{tabular}{ccccc}
\hline & \multicolumn{2}{c}{ OLTC } & \multicolumn{2}{c}{ Capacitor } \\
Scenario & $V_{\text {set }}(\mathrm{pu})$ & $V_{\mathrm{db}}(\mathrm{pu})$ & $t_{\mathrm{on}}$ & $t_{\mathrm{off}}$ \\
\hline \hline CC-A & 1.00 & 0.03 & $07: 00$ & $16: 00$ \\
\hline CC-B & 1.02 & 0.03 & $07: 00$ & $16: 00$ \\
\hline
\end{tabular}

Table 2

Optimum Settings

\begin{tabular}{cccccc}
\hline \multicolumn{2}{c}{ OLTC } & \multicolumn{2}{c}{ SC } & \multicolumn{2}{c}{ FCs } \\
$V_{\text {set }}$ & $V_{\mathrm{db}}$ & $Q_{\text {on }}$ & $Q_{\text {off }}$ & $V_{\text {on }}$ & $V_{\text {off }}$ \\
$(\mathrm{pu})$ & $(\mathrm{pu})$ & $(\mathrm{Mvar})$ & $(\mathrm{Mvar})$ & $(\mathrm{pu})$ & $(\mathrm{pu})$ \\
\hline \hline 1.035 & 0.03 & -2 & 2 & 0.99 & 1.05 \\
\hline
\end{tabular}

and improve the best solution by $1.02 \%$. Based on function evaluations of up to 1000 iterations, further increases in iterations do not lead to an improved best solution.

\subsection{Effect of Adaptive Reference Voltage and Tap Range}

Two cases consisting of 30 trials each are studied. The maximum number of iterations per trial is 200. In the first case, the reference voltage $V_{R}$ is fixed at 1 pu and the tap range is bounded by the highest and lowest possible transformer tap positions, $T^{\min }$ and $T^{\max }$ respectively. In the second case, the tap range and reference voltage are adjusted as defined in Section 3.3. Numerical results of the fitness function given by (13) are compared for both cases.

The best solution obtained with a fixed reference voltage and the full tap range, is a minimum of 2.3853. As mentioned previously, not all tap positions yield bus voltages between $V^{\min }$ and $V^{\max }$. As a result, the search space explored has a number of infeasible solutions. By comparison, the tap range and reference voltage adjustments improve the quality of solutions, with the best solution dropping to 2.3612. Similarly, the mean, among others is lowered to 2.4056 from 2.4225. A graphical description of the adapted tap range can be seen in Fig. 5. The solutions are improved in this case 
Table 3

Statistical results of FVC-OSC

\begin{tabular}{ccc}
\hline & FVC-OSC (Fixed) & FVC-OSC (Adaptive) \\
\hline \hline Worst & 2.5407 & 2.4633 \\
\hline Best & 2.3853 & 2.3612 \\
\hline Mean & 2.4225 & 2.4056 \\
\hline Standard Deviation & 0.0367 & 0.0249 \\
\hline
\end{tabular}

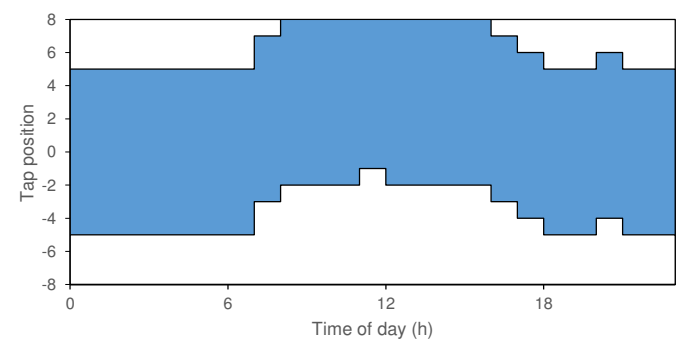

Fig. 5. Adaptive tap range.

because the algorithm explores a narrower search path defined by fewer tap positions. In other words, the algorithm is directed towards more feasible solutions within the preset maximum number of iterations. Details of the statistical results reached by the two cases are shown in Table 3 ,

\subsection{FVC-OSC and Conventional Control Comparison}

Table 4 shows the summarised results for the base case, conventional control and FVC-OSC. The purpose of the base case is to illustrate a system without VVC, in which the transformer tap position is fixed at nominal tap and the SC status is off. For conventional control, the voltage is kept within the specified deadband at bus 1 as illustrated in Fig. 6. On the contrary, the voltages at other buses fluctuate with load. Hence the lower voltage limit is violated at bus 9 and at bus 10 during the day for CCA. The voltage profiles for bus 9 are also shown in Fig. 6. The voltage remains within the allowable range throughout the day and the total daily loss is reduced in CC-B. This is due to the higher voltage set-point in this scenario. The total VDI decreases by $67.46 \%$ and the total loss by $1.07 \%$ from 0.1340 and 14.3422 MWh produced by 
CC-A respectively. It should be noted that raising the voltage set-point does not always improve the solution. For example, changing the set-point from $1.02 \mathrm{pu}$ to $1.03 \mathrm{pu}$ lowers the total loss to $14.0946 \mathrm{MWh}$ but increases the VDI to 0.0628. In FVC-OSC, the FCs remain switched on because the reactive power-flow at the either of the substation feeders does not exceed $-0.5 Q_{d}^{\mathrm{F}}$ at any instant during the $24-\mathrm{h}$ period. The resulting bus voltages are kept even closer to the desired values while there is also an improvement in the total loss reduction recorded in CC-B. Compared to CC-B, the VDI and the total loss are reduced by additional $13.99 \%$ and $1.47 \%$ respectively. FVC-OSC results correspond to a calculated reference voltage of $1.02 \mathrm{pu}$. For high load (07:00-18:00), FVC-OSC raises the voltage higher than in conventional control. The rest of the time, the voltage is maintained closer to CC-A or CC-B. This adaptive ability allows FVC-OSC to maintain a steadier voltage profile downstream in both high and light loading conditions. Bus voltages along the network are displayed in Fig. 7 These voltages are produced by conventional control and FVC-OSC when the load demand is at its maximum at 11:00. It can be seen that the voltage is closer to $1 \mathrm{pu}$ and more uniform for FVC-OSC than for CC-A and CC-B. The tap movements and capacitor control actions resulting from the cases under study are displayed in Fig. 8 , In all scenarios, the number of device operations remained below the specified limits although FVC-OSC resulted in the highest of all four. It can be observed that in FVCOSC, the OLTC raises the transformer tap position higher than in CC-A and CC-B, most notably at peak load. As expected, FVC-OSC produces the highest loss reduction during this period.

In the presence of load forecast errors, specifically between $-1 \%$ and $18 \%, \mathrm{FVC}-\mathrm{OSC}$ still keeps voltages within 0.95 and $1.05 \mathrm{pu}$. VDI is decreased by at least $18 \%$ and the loss is reduced by at least $1 \%$ from the conventional control case. Beyond this particular error range voltage limits are violated. For FVC-OSC to be able to maintain satisfactory performance under a larger error range, the model can be implemented with a feedback control technique. In this case a model predictive controller is a suitable candidate to act on load changes online in a manner akin to [Zhang, Xia]. 

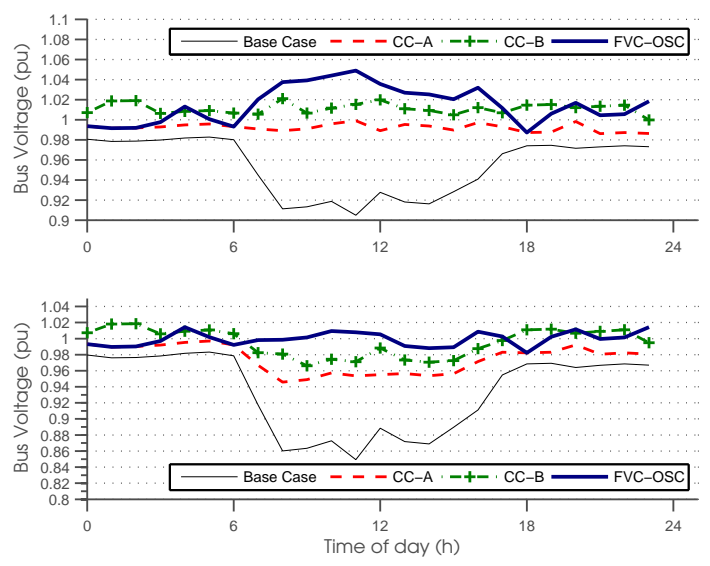

Fig. 6. Bus 1 (top) and bus 9 (bottom) voltage profiles.

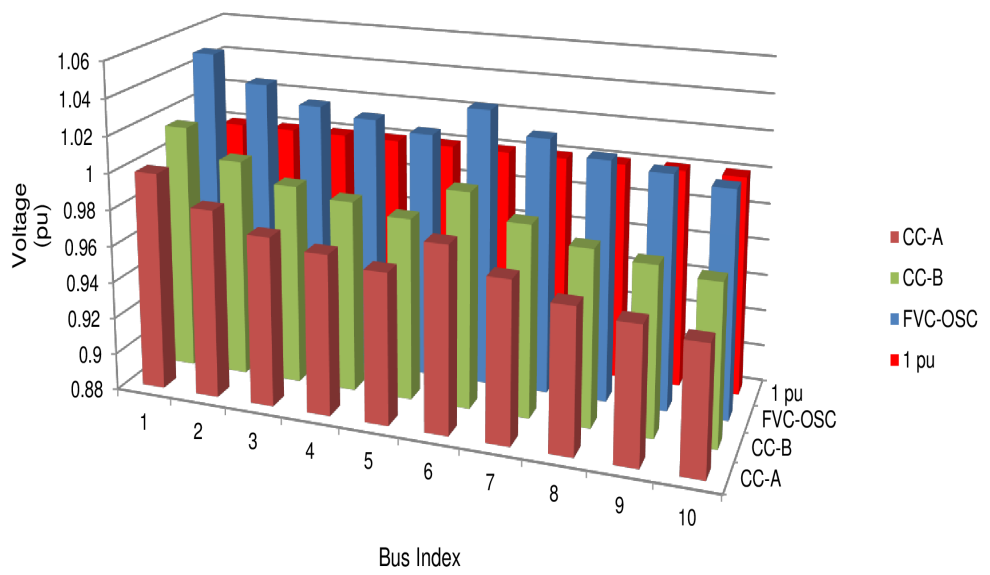

Fig. 7. Bus voltages produced by the different VVC approaches for maximum loading at 11:00.

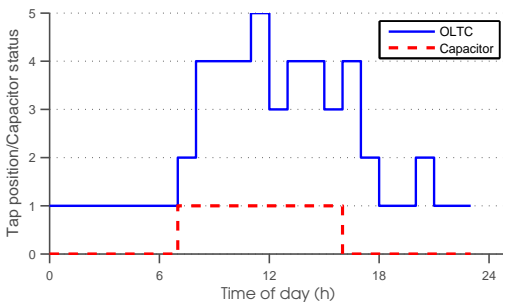

(a) CC-A

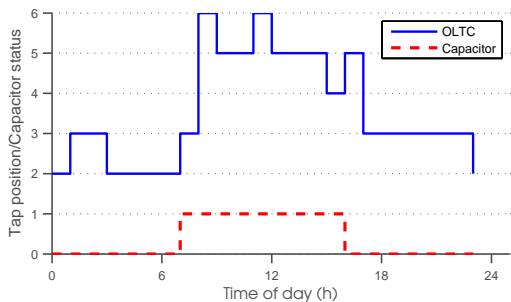

(b) CC-B

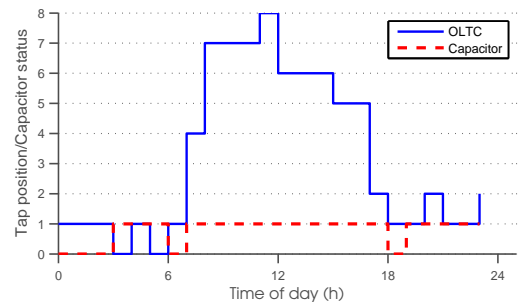

(c) FVC-OSC

Fig. 8. Simulated switching sequences of the substation devices over 24 hours. 
Table 4

Summary of results for the system under study

\begin{tabular}{ccccc}
\hline & & \multicolumn{3}{c}{$\begin{array}{c}\text { Number of } \\
\text { Operations }\end{array}$} \\
Tcenario & VDI & $\begin{array}{l}\text { Loss } \\
(\mathrm{MWh})\end{array}$ & OLTC & SC \\
\hline \hline Base Case & 1.2778 & 15.2520 & - & - \\
\hline CC-A & 0.1340 & 14.3422 & 14 & 2 \\
\hline CC-B & 0.0436 & 14.1888 & 14 & 2 \\
\hline FVC-OSC & 0.0375 & 13.9798 & 21 & 5 \\
\hline
\end{tabular}

\subsection{Effect of Limiting OLTC and Capacitor Operations}

As previously mentioned, the total number of operations produced by FVC-OSC is higher than that of conventional control. It is desirable to determine how the optimal solution is affected by the number of capacitor and OLTC operations. The relationship between the maximum allowed number of operations, the VDI and the total daily loss is shown in Table 5 and Table 6. For OLTC switching limits over 25, the number of feasible solutions increases together with the OLTC switching operations but, the best solution is not improved. The same pattern is displayed for capacitor control action limits over 6. For OLTC movements under 20 and capacitor control actions below 6, the VDI and the total loss increase as the number of operations drops. It is difficult for FVC-OSC to provide feasible solutions as the switching requirements become more stringent. In this case study, the algorithm cannot provide solutions which satisfy (8) and (4) when the limits on OLTC movements and capacitor operations are lower than nine and two respectively.

\subsection{FVC-OSC and OSA Comparison}

In this scenario, two more FCs are connected to bus 4 and bus 9 , resulting in four 1.4 Mvar FCs in the network. The voltage deviation trends for the optimum settings approach and FVC-OSC are shown in Fig. 9, The VDI is 0.0296 for FVC-OSC and 
Table 5

Effect of Limiting OLTC Operations

\begin{tabular}{ccccc}
\hline & & \multicolumn{3}{c}{$\begin{array}{c}\text { Actual Number of } \\
\text { Operations }\end{array}$} \\
Operations \\
$\begin{array}{l}\text { Limit } \\
\text { Total }\end{array}$ & VDI $\begin{array}{l}\text { Loss } \\
\text { (MWh) }\end{array}$ & OLTC & SC \\
\hline \hline 30 & 0.0485 & 13.9927 & 29 & 7 \\
\hline 25 & 0.0375 & 13.9798 & 21 & 5 \\
\hline 20 & 0.0385 & 14.116 & 18 & 4 \\
\hline 15 & 0.0367 & 14.1402 & 14 & 4 \\
\hline 10 & 0.0469 & 14.1558 & 9 & 4 \\
\hline
\end{tabular}

\section{Table 6}

Effect of Limiting Capacitor Switching Operations

\begin{tabular}{ccccc}
\hline & & \multicolumn{4}{c}{$\begin{array}{c}\text { Actual Number of } \\
\text { Operations }\end{array}$} \\
Operations \\
$\begin{array}{c}\text { Limit } \\
\text { Total }\end{array}$ & VDI $\begin{array}{l}\text { Loss } \\
\text { (MWh) }\end{array}$ & OLTC & SC \\
\hline \hline 10 & 0.0378 & 14.034 & 29 & 9 \\
\hline 8 & 0.0485 & 13.9927 & 29 & 7 \\
\hline 6 & 0.0375 & 13.9798 & 21 & 5 \\
\hline 4 & 0.0389 & 14.0773 & 26 & 4 \\
\hline 2 & 0.0405 & 14.1038 & 20 & 2 \\
\hline
\end{tabular}



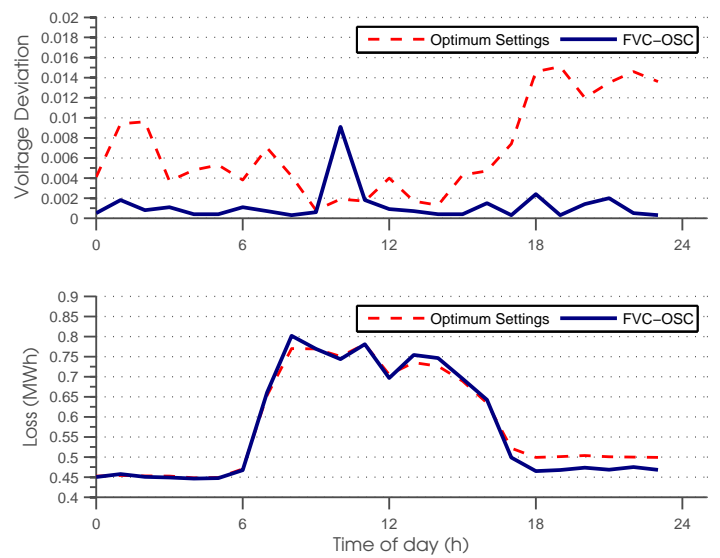

Fig. 9. 24-h voltage deviation (top) and loss (bottom) trends of FVC-OSC and the optimum settings method.

0.163 for the optimum settings approach. Both methods keep the voltage within upper and lower limits. The optimum settings approach maintains bus 1 voltage close to a predetermined set-point which, in this case, is largely in favour of loss reduction. Hence in this case study, the VDI is low for high loading but high for low loading. In contrast, FVC-OSC allows bus 1 voltage variations that are suitable for low losses and voltage deviations considering all the distribution buses.

The impact of the two different approaches on losses is also illustrated in Fig. 9. FVC-OSC produces losses equal to $13.7749 \mathrm{MWh}$ for the whole system and 2.4595 MWh for the distribution network. On the other hand, the optimum settings approach gives values of $13.9183 \mathrm{MWh}$ for the whole system and 2.6357 MWh for the distribution network. The lower loss magnitudes show the effectiveness of controlling the FCs based on reactive power set-points and incorporating the resulting switching sequences into the OSC model.

\subsection{9-bus System}

\section{Conclusion}

FVC-OSC has been presented with the purpose of dealing with daily VVC in a coordinated but simplified manner. The FCs are controlled according to reactive power-flows 
through distribution feeders while the OLTC and SC control problem is formulated with respect to operational constraints and then solved with discrete particle swarm optimisation. It is demonstrated that FVC-OSC determines the most suitable substation secondary bus reference voltage and dispatch sequences to minimise daily voltage deviations and total loss over 24 hours. This is in comparison with existing variants of conventional VVC. Results also show that FVC-OSC maintains feasibility even under tight restrictions on the allowable maximum number of control movements.

\section{References}

[1] T. A. Short, Electric Power Distribution Handbook, CRC, Boca Raton, FL, 2004.

[2] J. J. Grainger, S. Civanlar, Volt/Var Control on Distribution Systems with Lateral Branchces Using Shunt Capacitors and Voltage Regulators: parts I-III, IEEE Trans. Power App. Syst. PAS-104 (11) (1985) 32783297.

[3] L. Roytelman, B. K. Wee, R.-L. Lugtu, Volt/Var Control Algorithm for Modern Distribution Management System, IEEE Trans. Power Syst. 10 (3) (1995) 14541460.

[4] Z. Gu, D. Rizy, Neural Networks for Combined Control of Capacitor Banks and Voltage Regulators in Distribution Systems, IEEE Trans. Power Del. 11 (4) (1996) 19211928.

[5] J.-C. Wang, H.-D. Chiang, K. N. Miu, G. R. Darling, Capacitor Placement and Real Time Control in Large-Scale Unbalanced Distribution Systems: Loss Reduction Formula, Problem Formulation, Solution Methodology and Mathematical Justification, IEEE Trans. Power Del. 12 (2) (1997) 953958.

[6] A. Borghetti, Using Mixed Integer Programming for the Volt/Var Optimization in Distribution feeders, Elect. Power Syst. Res. 98 (8) (2013) 39-50.

[7] Y.-Y. Hsu, H.-C. Kuo, Dispatch of Capacitors on Distribution System Using Dynamic Programming, Proc. Inst. Elect. Eng., Gen., Transm., Distrib. 140 (6) (1993) 433-438.

[8] F.-C. Lu, Y.-Y. Hsu, Reactive Power/Voltage Control in a Distribution Substation Using Dynamic Programming, Proc. Inst. Elect. Eng. 142 (6) (1995) 639-645. 
[9] F.-C. Lu, Y.-Y. Hsu, Fuzzy Dynamic Programming Approach to Reactive Power/Voltage Control in a Distribution Substation, IEEE Trans. Power Syst. 12 (2) (1997) 681-688.

[10] Y.-Y. Hsu, F.-C. Lu, A Combined Artificial Neural Network-fuzzy Dynamic Programming Approach to Reactive Power/Voltage Control in a Distribution Substation, IEEE Trans. Power Syst. 13 (4) (1998) 1265-1271.

[11] R.-H. Liang, C.-K. Cheng, Dispatch of Main Transformer ULTC and Capacitors in a Distribution System, IEEE Trans. Power Del. 16 (4) (2001) 625-630.

[12] Y. Liu, P. Zhang, X. Qiu, Optimal Volt/Var Control in Distribution Systems, Elect. Power Energy Syst. 24 (4) (2002) 271-276.

[13] D. Youman, R. Xiaojuan, Z. Changcheng, Z. Dapu, A Heuristic and Algorithmic Combined Approach for Reactive Power optimization With Time-Varying Load Demand in Distribution Systems, IEEE Trans. Power Syst. 17 (4) (2002) 1068-1072.

[14] Z. Hu, X. Wang, H. Chen, G. A. Taylor, Volt/Var Control in Distribution Systems Using a Time-Interval based Approach, Proc. Inst. Elect. Eng. 150 (5) (2003) 548-554.

[15] R.-H. Liang, Y.-S. Wang, Fuzzy-Based Reactive Power and Voltage Control in a Distribution System, IEEE Trans. Power Del. 18 (2) (2003) 610-618.

[16] T. Senjyu, Y. Miyazato, A. Yona, N. Urasaki, T. Funabashi, Optimal Distribution Voltage Control and Coordination With Distributed Generation, IEEE Trans. Power Del. 23 (2) (2008) 1236-1242.

[17] M. B. Liu, C. A. Cañizares, W. Huang, Reactive Power and Voltage Control in Distribution Systems With Limited Switching Operations, IEEE Trans. Power Syst. 24 (2) (2009) 889-899.

[18] T. Senjyu, P. Mandal, K. Uezato, T. Funabashi, Next Day Load Curve Forecasting Using Hybrid Correction Method, IEEE Trans. Power Syst. 20 (1) (2005) 102-109.

[19] H. Kebriaei, B. Araabi, A. Rahimi-Kian, Short-Term Load Forecasting With a New Nonsymmetric Penalty Function, IEEE Trans. Power Syst. 26 (4) (2011) 1817-1825. 
[20] J.-Y. Park, S.-R. Nam, J.-K. Park, Control of a ULTC Considering the Dispatch Schedule of Capacitors in a Distribution System, IEEE Trans. Power Syst. 22 (2) (2007) 755-761.

[21] M. S. Calovic, Modeling and Analysis of Under-Load Tap-Changing Transformer Control 370 Systems, IEEE Trans. Power App. Syst. PAS-103 (7) (1984) 1909-1915.

[22] N. Yorino, M. Danyoshi, M. Kitagawa, Interaction Among Multiple Controls in Tap Change under Load Transformers, IEEE Trans. Power Syst. 12 (1) (1997) 430-436.

[23] F. A. Viawan, Voltage Control and Voltage Stability of Power Distribution Systems in the Presence of Distributed Generation, Ph.D. thesis, Chalmers University of Technology (2008).

[24] Y.-X. Jin, H.-Z. Cheng, J. Yan, L. Zhang, New Discrete Method for Particle Swarm Optimization and its Application in Transmission Network Expansion Planning, Elect. Power Syst. Res. 77 (3-4) (2007) 227-233.

[25] A. Bouhouras, G. Andreou, D. Labridis, A. Bakirtzis, Selective Automation Upgrade in Distribution Networks Towards a Smarter Grid, IEEE Trans. Smart Grid 1 (3) (2010) $278-285$.

[26] A. Onen, D. Cheng, R. Arghander, J. Jung, J. Woyak, M. Dilek, R. Broadwater, Smart Model Based Coordinated Control Based on Feeder Losses, Energy Consumption, and Voltage Violations, Elect. Power Compon. and Syst. 41 (16) (2013) 1686-1696.

[27] F. A. Viawan, D. Karlsson, Combined Local and Remote Voltage and Reactive Power Control in the Presence of Induction Machine Distributed Generation, IEEE Trans. Power Syst. 22 (4) (2007) 2003-2012.

[28] F. A. Viawan, D. Karlsson, Voltage and Reactive Power Control in Systems With Synchronous Machine-based Distributed Generation, IEEE Trans. Power Del. 23 (2) (2008) 1079-1087.

[29] R. D. Zimmerman, C. E. Murillo-Sánchez, R. J. Thomas, Matpower: Steady-State Operations, Planning and Analysis Tools for Power Systems Research and Education, IEEE Trans. Power Syst. 26 (1) (2011) 12-19. 